\title{
TV/Series
}

\section{« Tu n'as rien vu [en Irak] » : Logistique de l'aperception dans Generation Kill}

Sébastien Lefait

\section{(2) OpenEdition}

\section{Journals}

Édition électronique

URL : http://journals.openedition.org/tvseries/1248

DOI : 10.4000/tvseries. 1248

ISSN : 2266-0909

Éditeur

GRIC - Groupe de recherche Identités et Cultures

Référence électronique

Sébastien Lefait, « «Tu n'as rien vu [en Irak] » : Logistique de l'aperception dans Generation Kill », TV/ Series [En ligne], 9 | 2016, mis en ligne le 01 juin 2016, consulté le 01 mai 2019. URL : http:// journals.openedition.org/tvseries/1248; DOI : 10.4000/tvseries.1248

Ce document a été généré automatiquement le 1 mai 2019

\section{(c) (i) (9)}

$T V /$ Series est mis à disposition selon les termes de la licence Creative Commons Attribution - Pas d'Utilisation Commerciale - Pas de Modification 4.0 International. 


\title{
"Tu n'as rien vu [en Irak] »: Logistique de l'aperception dans Generation Kill
}

\author{
Sébastien Lefait
}

\section{Introduction : L'aporie de la fiction militaire}

1 La guerre, comme le rappelle Olivier Ammour-Mayeur, rend le témoignage à la fois indispensable et impossible ${ }^{1}$. Les conflits placent en effet le «témoin de l'indicible » dans une « situation aporétique ${ }^{2}$.» Pour Ammour-Mayeur, c'est ce que suggèrent les momentsclés du dialogue sur lequel s'ouvre un film de guerre à l'esthétique atypique, Hiroshima mon amour (Resnais, 1959), surtout si on le compare aux superproductions américaines de la décennie suivante. «Tu n'as rien vu à Hiroshima. Rien », déclare d'abord le personnage masculin, exprimant ainsi l'absence de preuve oculaire de l'événement sur laquelle fonder un témoignage. En effet, " pour avoir vu quelque chose, il aurait fallu être présent à Hiroshima le 6 août 1945 à 08h15. Or, ni lui ni elle ne s'y trouvaient ${ }^{3}$. " À quoi le personnage féminin, dont on apprendra plus tard qu'elle est une actrice venue jouer dans la reconstitution filmique des événements $\mathrm{du}$ second conflit mondial, rétorque abruptement : "J'ai tout vu. Tout », pour suggérer que la visualisation des faits peut se passer de témoin direct. Elle émet ainsi l'hypothèse d'une perception qui dépasserait les capacités de l'œil humain ${ }^{4}$, et mettrait fin à la situation aporétique. De fait, la sortie de l'impasse implique une forme de vision augmentée que seule la fiction audiovisuelle peut fournir. C'est ce qu'explique le personnage féminin, un peu plus loin dans le dialogue : «Les films ont été faits le plus sérieusement possible. L'illusion, c'est bien simple, est tellement parfaite que les touristes pleurent $»$.

L'aporie de la guerre indicible trouverait donc une solution dans «l'illusion parfaite » de la reconstitution à l'écran ${ }^{5}$. La fiction militaire a en effet pour vocation de mettre en récit cet inexprimable dont parle Ammour-Mayeur, mais également de rendre visible ce que le regard ne saurait soutenir. Dans cette configuration, l'absence de témoin direct est compensée par un effort d'imagination suivi d'une mise en images. Au terme de ce 
processus de reconstitution fictionnelle, l'événement est mis à distance, ce qui permet aux spectateurs de supporter la vision d'un conflit ainsi déréalisé.

3 Conçue par David Simon et Ed Burns d'après le roman éponyme d'Evan Wright (2004), Generation Kill, série en sept épisodes diffusée en 2008 sur HBO, montre que ce qui cause l'aporie du film de guerre a changé par rapport à l'époque d'Hiroshima mon amour. La raison principale en est que plus rien n'est invisible, et que la fiction militaire doit s'adapter à cet état esthétique sans précédent. Pour en souligner la nouveauté, la série narre le quotidien d'un bataillon de Marines durant la première campagne d'Irak, en 2003, tel qu'il est rapporté par un avatar fictionnel de Wright (Lee Tergesen). Ce faisant, elle place constamment entre les mains des personnages des dispositifs de capture d'images. L'aveuglement face à la réalité militaire placé au seuil d'Hiroshima mon amour laisse donc place à une hypervisibilité de la guerre, qui décuple a priori les possibilités d'en témoigner. Si l'aporie subsiste, elle ne saurait plus reposer sur l'absence de survivants ni sur le manque de documents. Le but est toujours de dévoiler les faits d'armes, mais le recours exhaustif à l'imagination ne se justifie plus.

4 Je propose de montrer ici en quoi le problème du témoignage impossible a évolué, et d'identifier les nouvelles ressources dont disposent les séries télévisées pour dire et pour montrer les conflits. Pour cela, j'appliquerai à la série de Simon et Burns les étapes du dialogue sur la Deuxième Guerre mondiale qui, dans Hiroshima mon amour, mène au constat d'impuissance, puis à son dépassement par la fiction. En effet, tout comme Hiroshima mon amour posait dans sa diégèse la question du témoignage et se posait ainsi, en tant que film sur la guerre, comme une réponse possible, Generation Kill montre que le problème de l'ineffable vient aujourd'hui d'un trop-plein d'images, et tente d'imposer, par son esthétique, une autre forme d'authenticité.

5 L'exemple que je privilégierai est celui de la séquence finale, où la série diffuse le reportage réalisé par l'un des Marines qu'elle a suivis pendant sept épisodes, le caporal Jason Lilley (Kellan Lutz). Grâce à son ordinateur portable, Lilley montre sa vidéo à l'ensemble de son bataillon sur le point de se séparer. Ce procédé de mise en abyme fait reposer le problème du film de guerre sur un décalage entre ce qui s'est passé en Irak et ce que les soldats en ont vu. C'est en fonction de ce hiatus, qui parcourt par ailleurs toute la série, que cette dernière invente une manière pertinente d'exprimer le conflit - en l'occurrence l'opération Iraqi Freedom de 2003. Pour en comprendre le fonctionnement, il faut donc caractériser cette faille ontologique entre l'événement et sa perception, et examiner son impact sur la représentation du conflit irakien.

\section{1. « J'ai tout vu. Tout. » Guerre et logistique de la perception}

6 La séquence finale prend la forme d'un document censé être authentique. Il s'agit en effet du film amateur réalisé par l'un des Marines, qui propose ici une prise de vue des opérations militaires au quotidien. Placée à la toute fin de la série, cette vidéo imbriquée atteste d'une inversion par rapport à l'absence de témoin direct qui suscitait l'aporie chez Resnais. À cause de l'omniprésence des dispositifs de capture audiovisuelle, le témoignage par l'image n'est plus absent, mais surabondant jusqu'à l'obscène des plans de cadavres qui figurent dans le pot-pourri audiovisuel de Lilley. Il est, de plus, disponible très rapidement, et diffusable très facilement : Lilley a vraisemblablement utilisé un logiciel 
de montage pour mettre bout-à-bout des extraits des séquences qu'on l'a vu tourner tout au long de la série, ce qui lui permet de montrer son reportage immédiatement après la fin des hostilités. On imagine que, son film étant stocké sur son ordinateur personnel, il n'aura aucun mal à l'envoyer sur Internet. Si le conflit est immontrable, ce n'est donc plus à cause d'une impossibilité de voir que vient nier une technologie optique ubiquitaire ${ }^{6}$.

\section{Le cyborg impuissant, ou la prothèse à l'origine du handicap}

7 En effet, comme l'a montré Paul Virilio, la guerre repose depuis le premier conflit mondial sur une « véritable logistique de la perception militaire ${ }^{7}$. " Virilio entend par là que « la représentation des événements domine la présentation des faits ${ }^{8}$. » Generation Kill met en scène cette primauté des technologies visuelles sur la perception directe, qui induit la possibilité de récolter quantité d'images témoin (Fig. 1).

Fig. 1 - La guerre au prisme de l'objectif

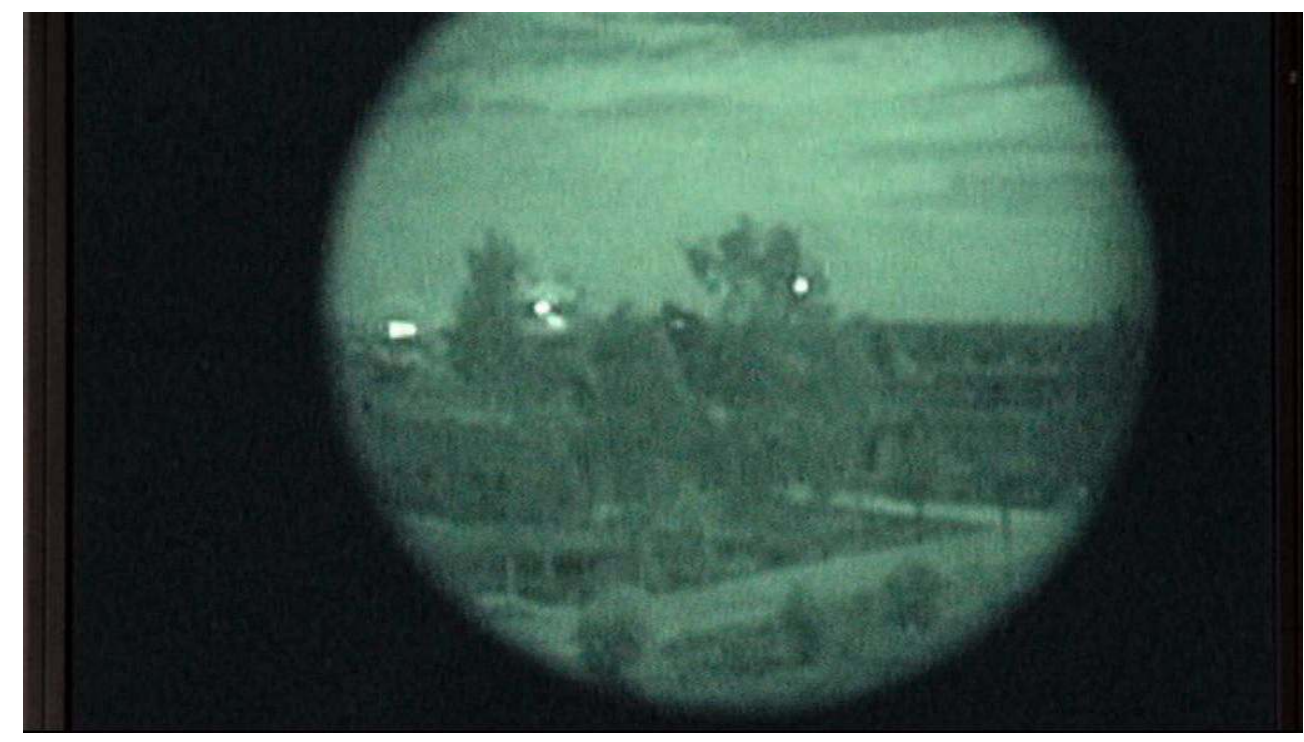

Pour le souligner, la série montre en permanence les soldats affublés des prothèses oculaires qui font aujourd'hui partie de leur attirail. C'est ce qu'illustrent, dans la séquence finale, les nombreux plans qui mettent en place une ocularisation interne primaire, signifiant au spectateur que ce que voient les militaires passe par un appareil de perception à distance, que ce soit le viseur d'un bazooka ou un dispositif de vision nocturne ${ }^{9}$. La disponibilité presque invasive de la technologie induit ainsi une volonté de surpuissance qui ne se traduit plus seulement par une hypertrophie musculaire signe de force (Fig. 2), mais aussi par des appendices télescopiques et phalliques qui lient le pouvoir à la possibilité de tout voir (Fig. 3). 
Fig. 2 - Des cyborgs impuissants...

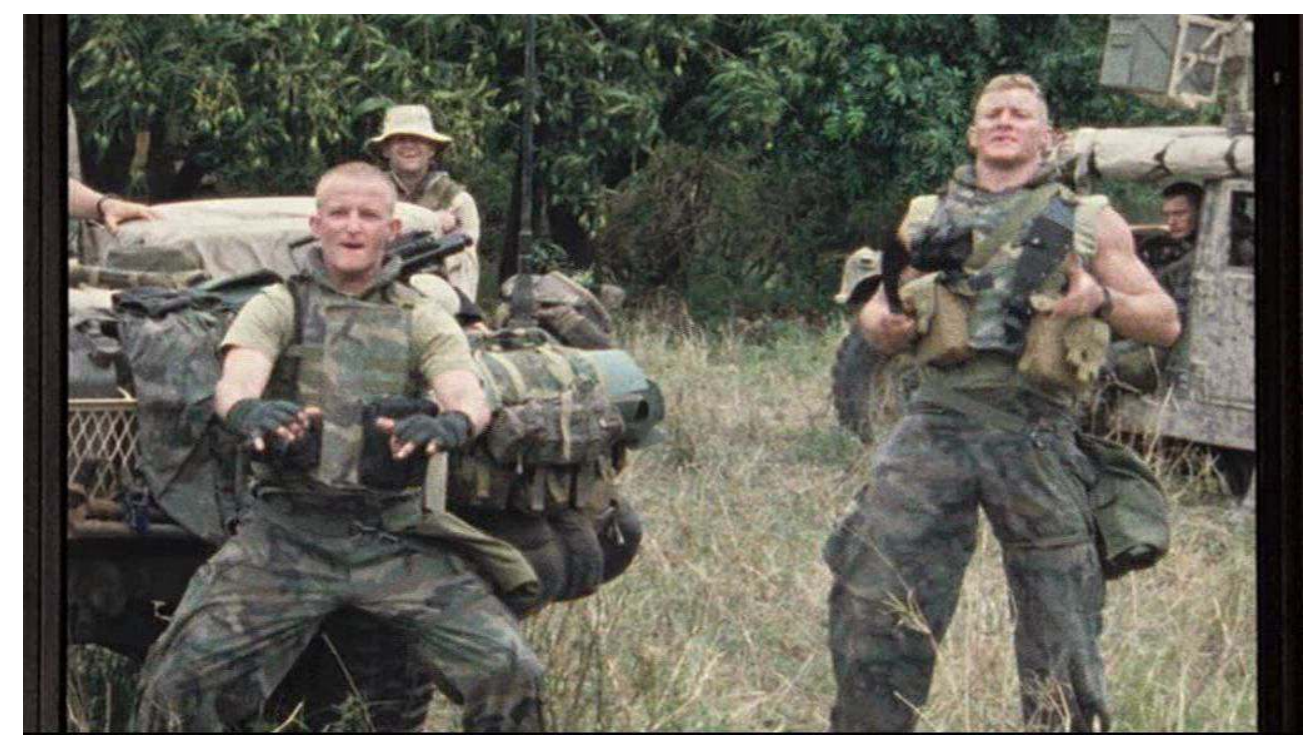

9 À de nombreuses reprises, les Marines laissent même échapper que l'outillage visuel fait quasiment partie d'eux-mêmes, à tel point qu'ils ne peuvent plus s'en passer. Ainsi, la sous-alimentation des dispositifs de vision nocturne, qui souffrent d'un manque chronique de batteries, fait l'objet de tant de protestations qu'elle apparait comme un véritable handicap. Privés de ces instruments, les soldats se sentent comme amputés d'un organe.

Fig. 3 ... à la virilité prosthétique

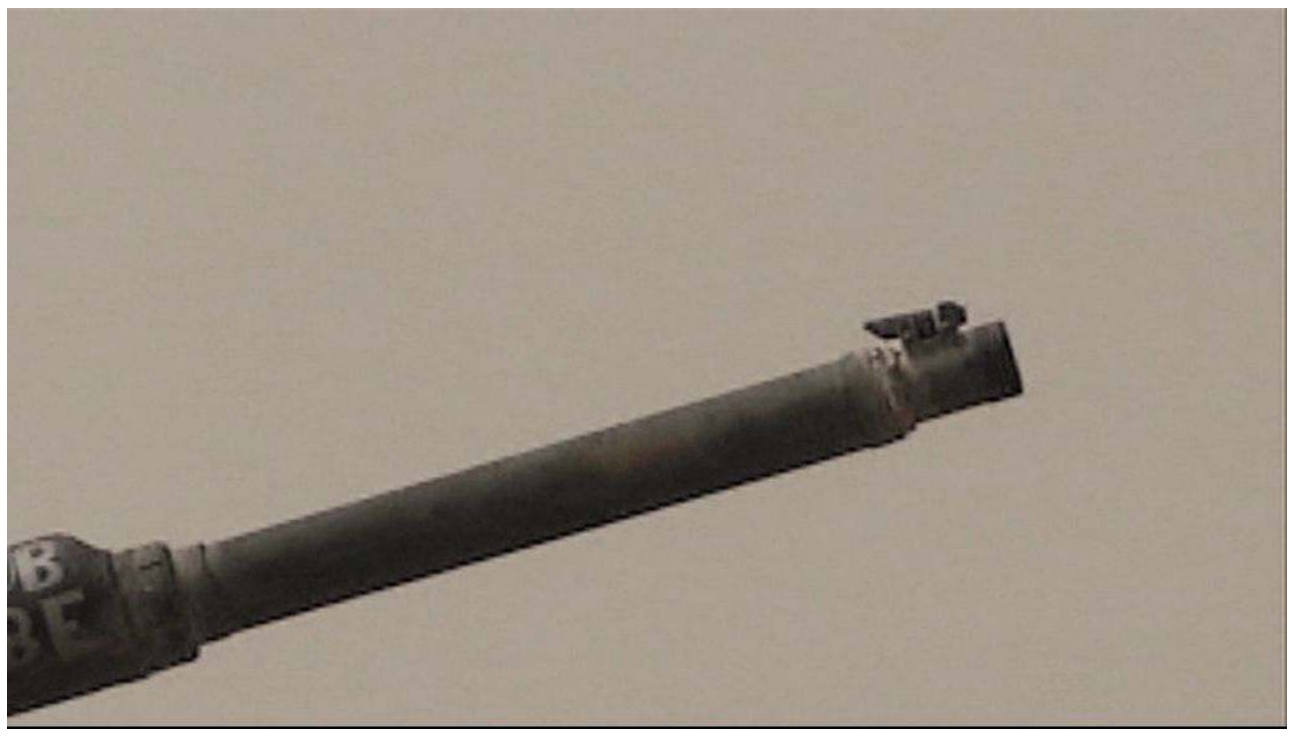

Mais tant qu'ils sont «appareillés », même la nuit ne cache plus rien pour ces cyborgs guerriers ${ }^{10}$. L'intensité hors normes de leur regard pénétrant fait donc que le théâtre des opérations, cartographié ${ }^{11}$ et passé au peigne fin des radars, n'a plus de secret pour personne. C'est ce que rappellent ici les plans d'hélicoptères en vol qui, évoquant la « mort venue du ciel » d'Apocalypse Now (Coppola, 1979), suggèrent qu'elle passe d'abord aujourd'hui par une vision surplombante et globale des territoires. La volonté de 
puissance implique alors de devenir une machine (à tuer), comme le montre ironiquement le plan où Colbert (Alexander Skarsgard), tel un enfant, joue à faire l'avion.

\section{La guerre comme art de la débuscade}

11 La motivation principale des soldats est ce fantasme d'une vision décuplée que traduit une relation érotique à leur arme - protubérance impuissante sans son indispensable viseur $^{12}$. Leur jouissance provient de la sensation de percer l'interdit, de voir ce qui devrait rester caché $^{13}$. Le contenu même du film de Lilley documente cette obsession de tout montrer, puisqu'on y voit les soldats filmés dans des endroits qu'ils traitent comme s'il s'agissait de lieux touristiques (Fig. 4).

Fig. 4 - J'ai tout vu : j'y étais

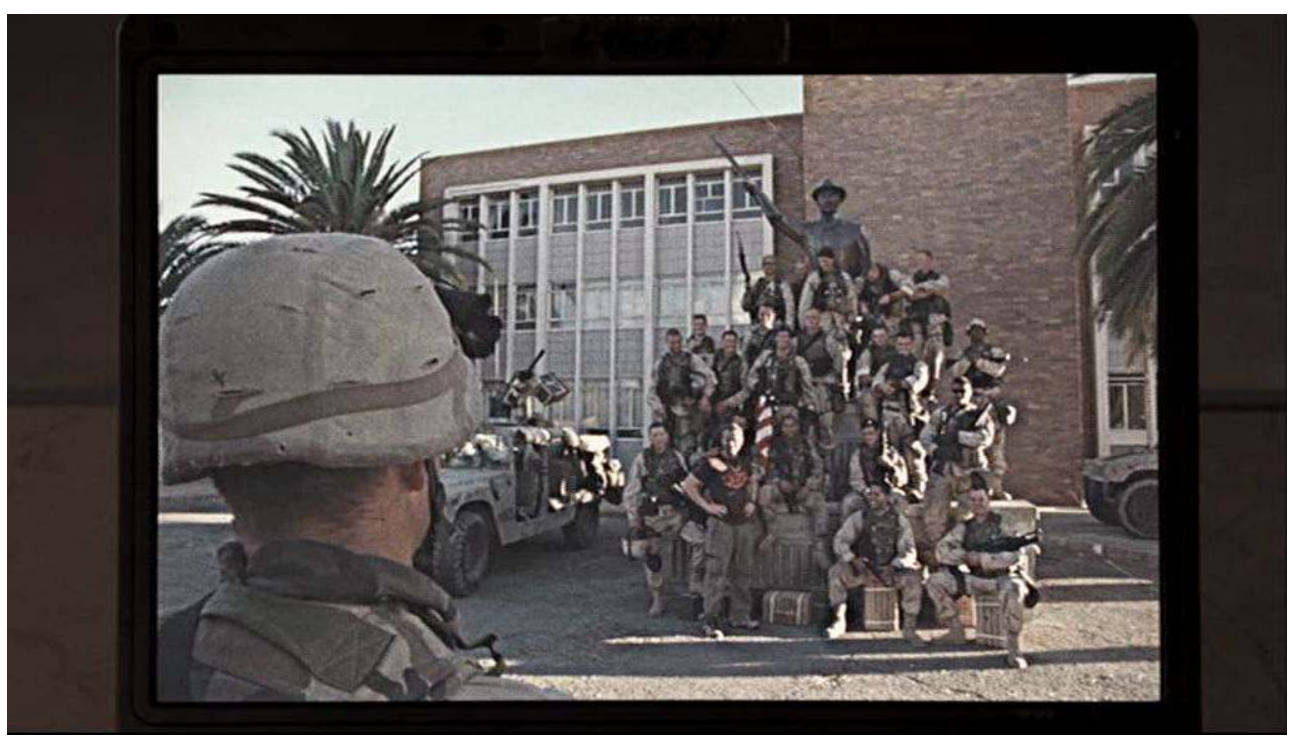

12 En guise de reportage sur les conflits filmés "de l'intérieur », les spectateurs assistent à une mise en spectacle du quotidien. Le film amateur qui tient lieu de séquence finale égrène en effet les « selfies » par lesquels les soldats entendent prouver leur présence sur les lieux des opérations militaires. 
Fig. 5 - Selfie : Rudy et un enfant irakien

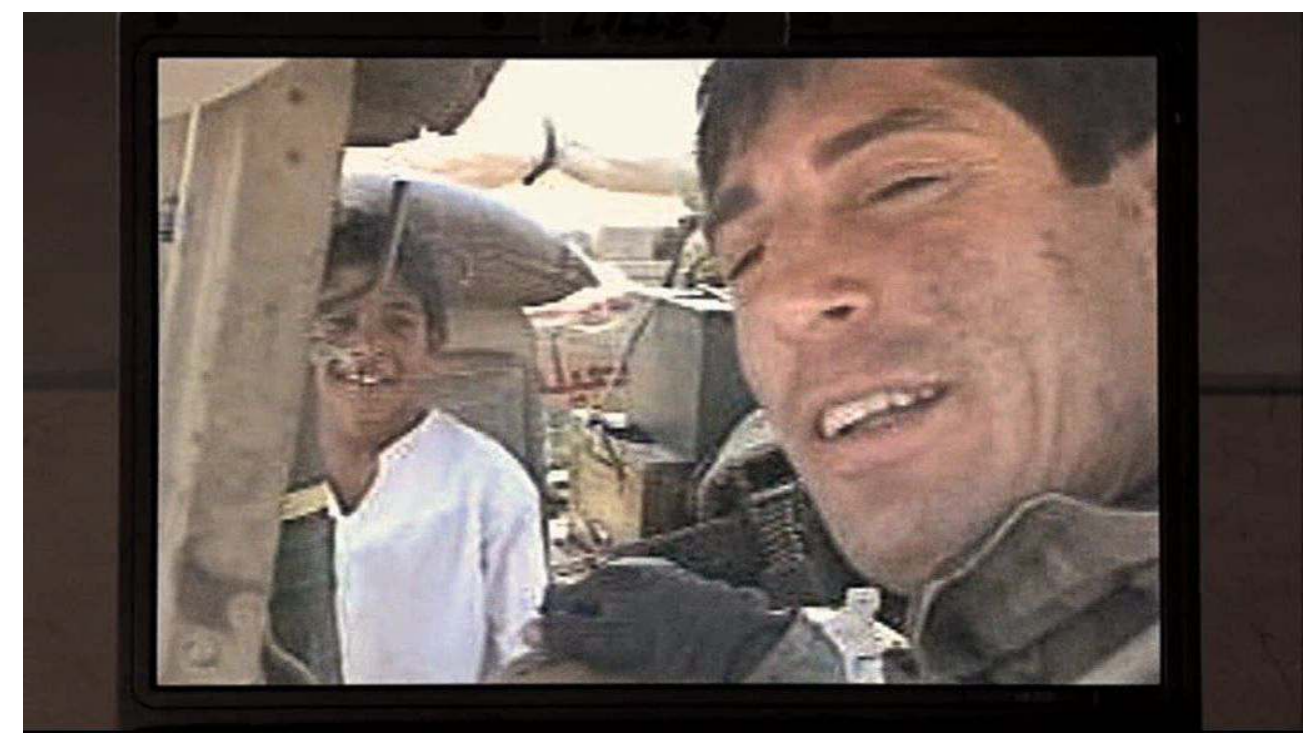

Ces autoportraits font de l'Irak un simple arrière-plan, qui se voit donc privé de la dimension concrète indispensable pour que soient exprimées l'horreur et la violence des combats (Fig. 5 et Fig. 6).

Fig. 6 - Selfie : Colbert et Person

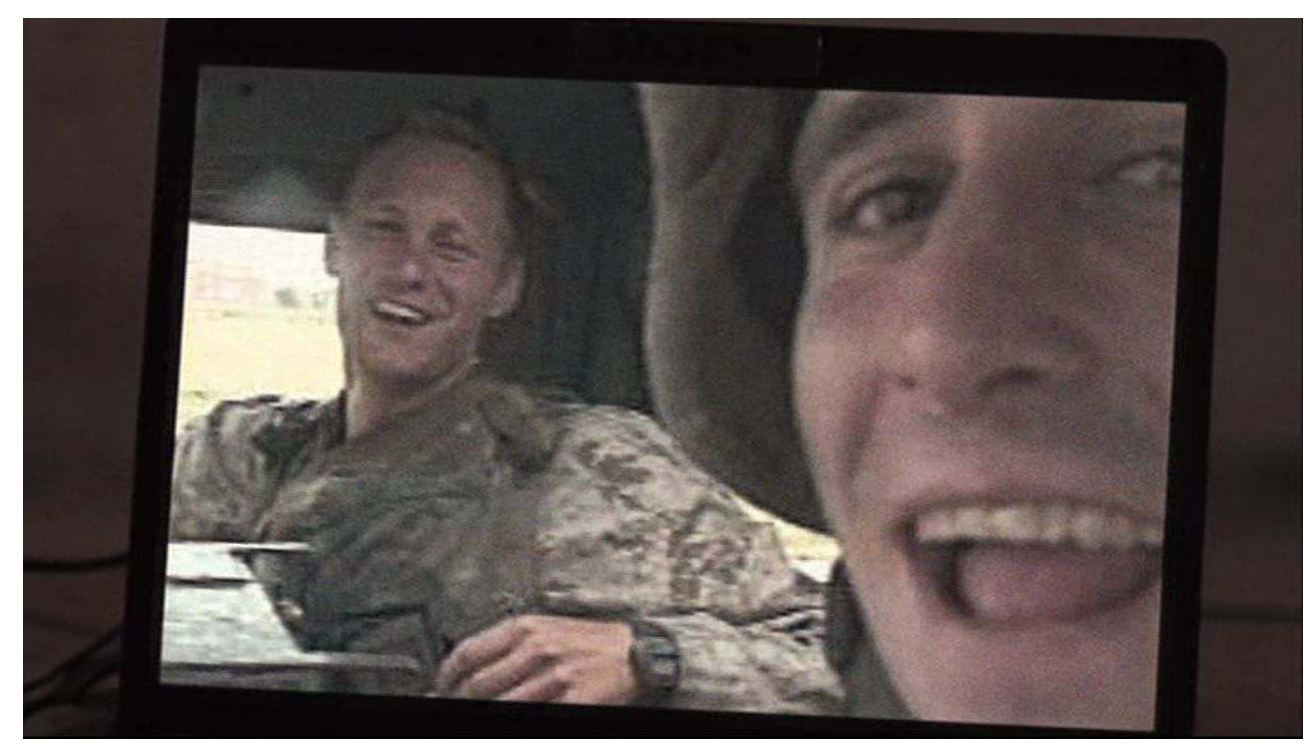

Dans ce contexte scopique propre aux guerres modernes, on voit, mais on voit de loin, et quand on tire, c'est sur une image. L'omniprésence des appareils de vision met en effet le réel à distance, transformant la guerre en art de la débuscade parce que la machine de guerre est devenue une «machine de guet ${ }^{14}{ }^{4}$. Dans ces conditions, repérer l'ennemi, c'est déjà le vaincre, et la seule muraille que l'on cherche à percer est la surface des apparences ${ }^{15}$. Pour cette raison, la séquence finale se résume à une accumulation de plans de visée, mis bout à bout avec des plans où l'on voit un projectile atteindre sa cible, sans lien de causalité apparente entre le tir et l'explosion. À la suite de quoi, seuls les soldats dotés d'une conscience vont constater de leurs yeux qu'ils ont abattu un combattant ennemi ou un civil. Ce qui compte avant tout, c'est la détonation du tir qui atteint sa cible, preuve 
de la précision chirurgicale des armes employées, et source de cris d'enthousiasme chez les soldats rassemblés autour de l'ordinateur pour visionner la vidéo de Lilley.

\section{Guerre et délocalisation de la violence}

Cette prédominance des plans où un projectile touche au but à l'échelle de la séquence rappelle donc que la logistique de la perception dont parle Virilio a pour but de déréaliser l'acte guerrier en en gardant le résultat concret à distance de son auteur ${ }^{16}$. Par ailleurs, toujours selon Virilio, les conflits modernes font primer le temps sur l'espace. Les soldats doivent agir vite et réagir dans l'urgence, pour avoir un coup d'avance sur l'ennemi dans le jeu guerrier dont ils sont les pions. Le corrélat de cette vitesse est la négation de la distance, sur laquelle la série insiste en montrant que, dans le viseur des Marines, le proche se confond avec le lointain ${ }^{17}$. La précision extrême des armes permet en effet d'atteindre des cibles très éloignées. C'est ce qui explique que le sergent Steven Lovell qualifie un tir d'artillerie tombant à moins de 600 mètres d'une position amie de "dangereusement proche ${ }^{18}$.»

Que les soldats suivis par la série fassent partie d'une escouade de reconnaissance prend alors tout son sens. Leur mission est réduite à néant, puisque le territoire à envahir est déjà couvert, passé au crible du radar ou du satellite ${ }^{19}$. À travers les étapes de leur parcours, il ne leur reste plus qu'à reconnaître un terrain connu, à voir de plus près ce qu'ils ont déjà vu de loin. Mais dans ce cas, pourquoi parcourir des kilomètres que la vitesse contracte? Sur ce point, la réponse de la série est assez claire. Progresser, c'est aller voir de ses propres yeux ce qu'on a d'abord vu par le petit bout de la lorgnette, et constater les dégâts provoqués par les tirs lointains. En avançant vers Bagdad, les Marines de la compagnie Bravo Two vont de cible en cible, mais n'atteignent chacune que pour que la suivante soit à portée de canon.

17 Paradoxe de cette abondance de repères produits par la machine de vision guerrière, les soldats ne savent ni où ils sont, puisqu'ils craignent de prendre une école pour une cible légitime, ni ce qu'ils font au moment de leurs actes, ne découvrant qu'après coup si, en tirant, ils ont éliminé un ennemi ou assassiné un civil. Peu importe, puisque l'acte, accompli dans un non-lieu, $u$-topie immatérielle d'une guerre sans conséquences, ne peut se solder que par un jugement de non-lieu. Désinhibés par cette mise à distance permanente de la réalité guerrière, par cette délocalisation de la violence, certains soldats, à l'image de Trombley (Billy Lush), n'ont qu'une hâte : celle de pouvoir abattre un être vivant, fût-ce un chien ${ }^{20}$.

Ironie suprême, cependant : dans ce nouveau rivage des Syrtes qu'est l'Irak de Generation Kill, les occasions de se décharger émotionnellement en appuyant sur la détente se font attendre et, lorsqu'elles se présentent, mènent à l'insatisfaction. La meilleure preuve en est le match de football américain improvisé qui précède la séquence finale (Fig. 7). 


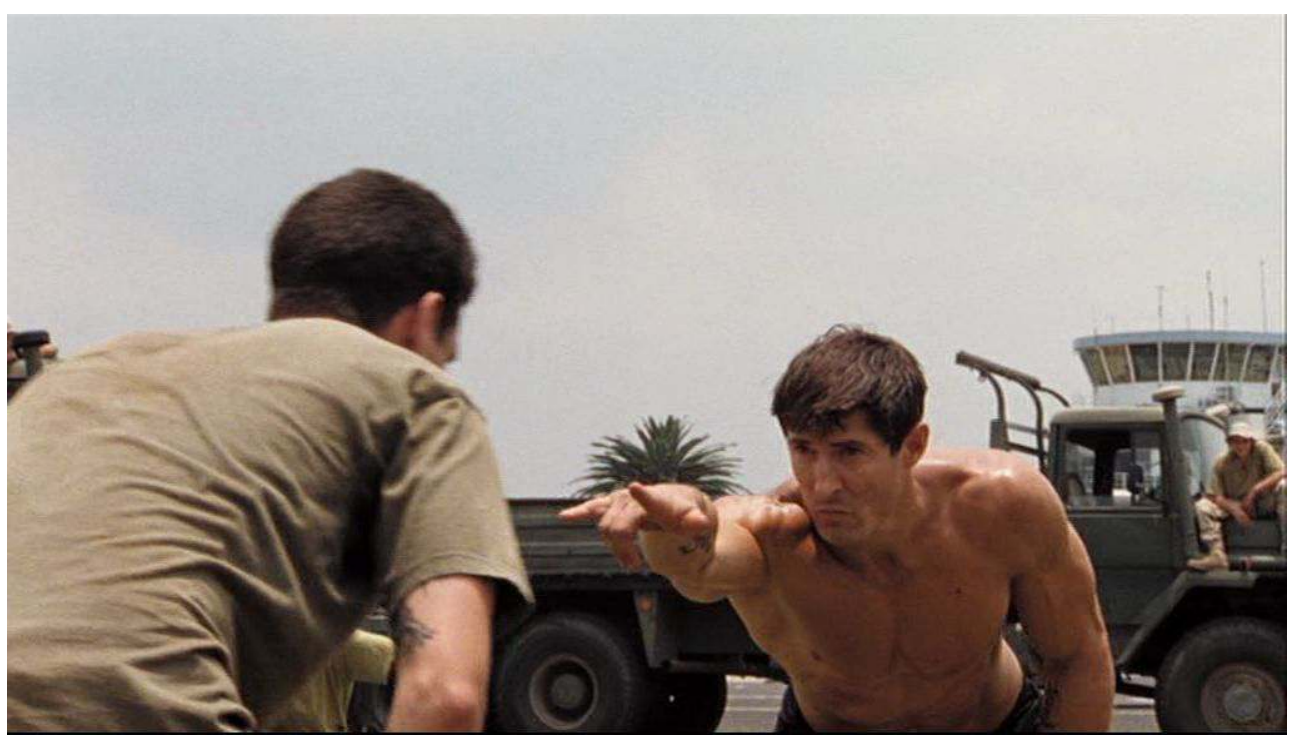

Par deux fois au cours de la partie, le jeu dégénère en pugilat entre Marines qui, compatriotes et compagnons d'armes quelques instants auparavant, semblent saisir l'occasion de se battre enfin contre quelqu'un. Au-delà du fait qu'elle contredit l'atmosphère de franche camaraderie qui émane du film amateur de Lilley, cette rencontre sportive qui dégénère alors que les tirs ennemis sont éteints révèle que les soldats ont accumulé une tension et une haine qu'ils n'ont pu libérer au combat. On peut y voir la preuve que le conflit irakien s'est terminé avant même d'avoir commencé, générant chez les militaires un état de manque face à une guerre qui a peut-être eu lieu, mais qu'ils ne semblent pas avoir vécue. C'est ce que montre ironiquement cette scène où le sport devient cathartique, et l'affrontement ludique plus intense que le conflit armé. Il apparaît ainsi que la logistique de la perception a pour pendant une logique de la frustration.

\section{2. «Tu n'as rien vu [...]. Rien. » Logistique de l'aperception, ou le « déni de l'image pleine ${ }^{21}$ "}

Par cette accumulation d'images découplées de leur référent, de conséquences que la capture vidéo dissocie de leurs causes, la séquence suggère donc que, malgré leur situation d'hypervision, les soldats n'ont rien vu. De manière plus polémique, elle montre même, comme toute la série, que la technologie, en donnant aux soldats l'obligation de mieux voir, les empêche de s'apercevoir de ce qu'ils font ${ }^{22}$. C'est par ce biais qu'elle induit une réflexion sur la manière dont les conflits contemporains s'appuient sur une culture visuelle fondamentalement différente de ce qu'elle était à l'époque d' Hiroshima mon amour

\section{Aux limites de l'hypervigilance}

21 En effet, l'omniprésence des appareils optiques dans Generation Kill ne vise pas tant à montrer la prédominance des représentations sur les faits qu'à dévoiler la face cachée du dispositif des guerres modernes, en montrant qu'elles comptent de plus en plus sur une 
logistique de l'aperception - terme dans lequel le préfixe est privatif. L'association entre le regard des spectateurs et celui des personnages permet en effet d'envisager les modalités d'un dévoilement au terme duquel la série nous « ouvre les yeux», paradoxalement, sur la manière dont la technique nous empêche de voir (Fig. 8).

Fig. 8 -Tu n'as rien vu. Rien.

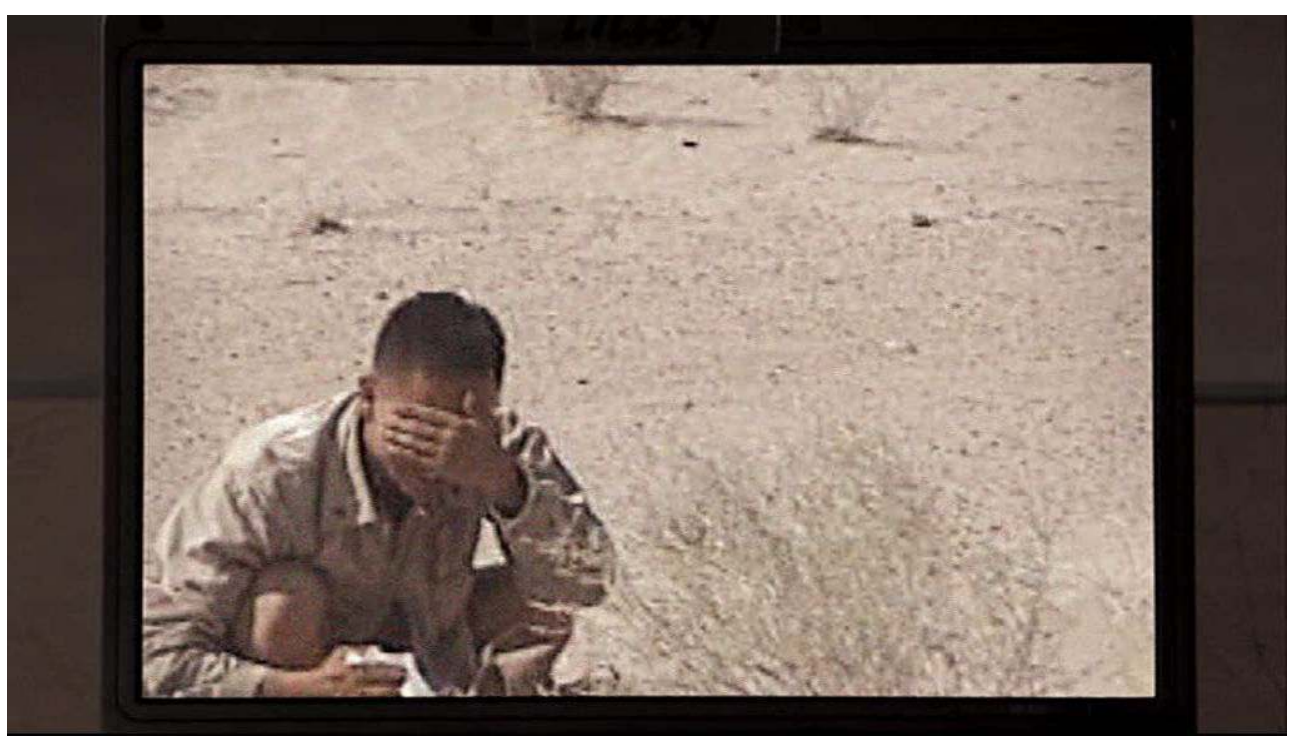

C'est ce qui ressort des nombreux moments où elle nous restitue ce que voient les soldats, pour qui l'adversaire irakien est au mieux aperçu, et au pire a-perçu. Ainsi, tout au long de Generation Kill, les divers instruments qui équipent les Marines leur font percevoir à distance un adversaire avec qui l'affrontement ne peut être qu'indirect. L'ennemi se fait attendre puis, lorsqu'arrive enfin le moment de l'assaut, demeure impalpable, immatériel. S'ajoutent à cela privation de sommeil et surconsommation de stimulants, qui expliquent que les soldats perçoivent la réalité de manière déformée. Dans le pire des cas, ils souffrent même d'hallucinations que les plans subjectifs filtrés par les prothèses oculaires permettent de visualiser, tant tout y paraît sans profondeur, monochromatique, ou flou.

Pour renforcer cette impression, la série montre également, dans ses nombreuses séquences de nuit, qu'une telle hypervision ne saurait être que relative. L'obscurité y est en effet tellement profonde que les spectateurs n'y voient presque rien. En comparaison, les plans subjectifs qui rendent la perception à travers les lunettes de vision nocturne paraissent nets. À y regarder de plus près, cependant, on s'aperçoit qu'un tel constat résulte d'une illusion d'optique due à la succession de plans opaques et d'images plus nettes. Le regard amplifié ne perçoit que des formes mouvantes, sans profondeur et sans couleurs, et ne bénéficiant donc que d'une relation indicielle très ténue avec le réel.

Autre signe de dysfonctionnement d'une technologie par ailleurs adulée: ce déficit perceptif entretenu implique une recherche frénétique de repères. Celle-ci prend une dimension absurde quand on envisage de donner des fanaux à des collaborateurs vivant dans le village ennemi, pour qu'ils "marquent» à distance les véritables méchants à abattre. À cet ordre, l'un des soldats rétorque en effet qu'on ne pourra pas déterminer si l'Irakien ainsi missionné par le camp adverse n'a pas désigné une personne comme cible simplement parce qu'elle lui devait de l'argent. Au lieu de la transparence recherchée, le stratagème ne fait donc que brouiller - un peu plus - les pistes. 


\section{La guerre-jeu vidéo : subjectivité par avatar interposé et illusion d'une "surcharge sensorielle ${ }^{23}$ "}

25 Absents à eux-mêmes malgré leur perception décuplée, les Marines semblent donc n'avoir rien vu d'autre en Irak qu'un de ces films de guerre auxquels ils font sans arrêt référence. Ils n'ont rien ressenti d'autre, pour certains, que le frisson jouissif bien connu du gamer patenté qui s'adonne au shoot'em up ${ }^{24}$. En effet, ainsi que l'a montré Christian Salmon ${ }^{25}$, une nouvelle ère s'est ouverte pour le mélange entre fiction et réalité quand l'armée américaine a fait concevoir des jeux vidéo pour faciliter la préparation de ses troupes. L'entraînement militaire moderne utilise les techniques les plus abouties de réalité virtuelle dans le cadre de jeux qui simulent les affrontements.

La conséquence de cette nouvelle approche des combats est double. Tout d'abord, l'initiation à la guerre par jeu vidéo interposé identifie le joueur à un avatar selon un processus qui perdure quand ledit joueur devient soldat. Pour dénoncer cette forme d'endoctrinement, Generation Kill met en scène des Marines qui se complaisent dans un rôle caricatural de guerriers sanguinaires, ce qui leur ôte toute culpabilité. Ainsi, dans une séquence marquante du premier épisode, Person (James Ransone) imagine à voix haute ce qu'il pourrait répondre à la lettre d'un écolier nommé Frederick, qui écrit pour témoigner son soutien aux soldats. Il proclame alors sur un ton hyperbolique que, quand on va à la guerre, c'est évidemment pour se mettre dans la peau d'un tueur sans merci.

PERSON. Dear Frederick! Thank you for your nice letter. But I am actually a US Marine who was born to kill, whereas clearly you have mistaken me for some sort of wine-sipping communist dicksuck. And although peace probably appeals to treeloving bisexuals like you and your parents, I happen to be a death-dealing, bloodcrazed warrior who wakes up every day just hoping for the chance to dismember my enemies and defile their civilizations. Peace sucks a hairy asshole, Freddie. War is the motherfucking answer.

Dans le même ordre d'idées, la série montre qu'il existe une continuité entre ce mode d'identification qui rend la guerre ludique et l'identification, plus classique, à des personnages fictionnels. Ainsi, les soldats ont tous un surnom, la plupart du temps emprunté à la culture de masse ${ }^{26}$. Les Marines perdent leur identité en entrant dans l'armée, pour endosser un rôle valorisant et connu, signe qu'ils participent à une action collective et ne sont pas individuellement responsables des exactions qu'ils commettent. Pour souligner cette caractéristique des soldats, la série joue sur son absence assumée de musique extradiégétique. Pour compenser ce manque d'une bande-son, les Marines fournissent régulièrement l'ambiance musicale qui leur parait adéquate en chantant à voix nue. Ils révèlent ainsi leur conviction de pouvoir vivre le conflit irakien comme s'il s'agissait d'une fiction cinématographique ou télévisée, en comblant au niveau diégétique les vides de l'univers fictionnel qui les héberge. Ce comportement rappelle que, comme le jeu vidéo, la fiction audiovisuelle impose aux combattants un filtre perceptuel qui esthétise et dédramatise souvent la violence - dans tous les sens du terme ici, puisqu'elle intervient hors de tout cadre logique ou narratif. La modalité fictionnelle applique ainsi une distance lénifiante à la barbarie militaire.

Pour relativiser l'ampleur de ce discours critique induit par la série, on pourrait objecter que Salmon déplorait déjà, il y a bientôt dix ans, que le régime fictionnel du jeu vidéo soit transposé dans la réalité des combats ${ }^{27}$. Dans son analyse du rôle déculpabilisant de divers types de médias, la série va cependant plus loin que cette dénonciation des enjeux 
idéologiques de l'identification. Elle montre en effet que l'action par personnage interposé induit une modification du degré d'objectivité associé à la perception. Or, ce transfert perceptif du médiatique virtuel au réel agit à une échelle bien plus importante que s'il se limitait à un simple phénomène d'identification. On n'encourage pas simplement les soldats à reproduire dans la réalité des réflexes acquis dans un univers virtuel. Ce faisant, on les invite également à transposer une vision immatérielle du monde sur la réalité. En effet, l'un des buts principaux des jeux en question est de préparer les guerriers aux conflits modernes, où l'ennemi est noyé dans la masse, invisible, prêt à surgir à n'importe quel moment. Les jeux sont donc censés augmenter la capacité perceptive des soldats, pour développer leur capacité de réaction. En d'autres termes, il s'agit de débusquer l'ennemi assez tôt pour qu'il ne puisse vous abattre avant que vous ne l'abattiez ${ }^{28}$. Le jeu vidéo doit donc induire un régime scopique de l'hypervigilance, et les autorités militaires espèrent que ce point de vue médiatisé sera transposé par les soldats dans le monde réel.

Par de nombreux aspects, Generation Kill met en scène cette dérive. La série montre d'une part qu'un tel transfert de régime scopique est d'autant plus aisé que, dans le contexte des conflits modernes, la perception du réel est, elle aussi, médiatisée. Elle montre d'autre part que ce transfert s'appuie sur une culture visuelle en profonde mutation, ce qui fera l'objet d'un développement plus détaillé ci-après.

\section{Profusion d'images, perte de la syntaxe, et prédominance du témoignage narcissique}

La réflexion sur cette évolution que subit notre perception justifie, dans la séquence finale, le recours à la mise en abyme. Comme dans le cas précédemment évoqué des plans de nuit mis en regard de ce que perçoivent les appareils de vision nocturne, le procédé permet de placer côte à côte ce que l'on pouvait voir - rendu par le contenu de la vidéo, qui montre ce que Lilley a retenu du conflit - et ce qu'il y avait effectivement à voir en Irak. De toute évidence, les Marines n'ont pas tous vu les mêmes événements, et il revient à la série de montrer la guerre de manière plus exhaustive ${ }^{29}$. Ainsi suggérée, la possibilité d'une autre version de la guerre d'Irak est également transcrite dans les réactions diversifiées des spectateurs assemblés autour de l'ordinateur portable. Person et Colbert échangent des regards complices qui montrent bien que la vidéo ne correspond pas à leur vision des choses. À l'inverse, Trombley, que l'on a vu tout au long de la série tuer pour le plaisir comme s'il jouait à un jeu vidéo, semble apprécier énormément le film amateur. Il reste jusqu'au bout face à l'écran, alors que la plupart de ses camarades s'éloignent au moment où les images leur rappellent l'atrocité de leurs actes. La fin du récit permet ainsi de distinguer ceux qui, comme Person et Colbert, ont été capables de reconnecter l'image de leurs agissements avec la réalité, de ceux qui, comme Trombley et Lilley, en sont restés au stade de la distanciation par l'image, et continuent à bénéficier de sa fonction déculpabilisante (Fig. 9). Ainsi, Trombley ne semble se rendre compte à aucun moment de la gravité du film de Lilley, qui exprime la « vision sans regard » dont parle Virilio ${ }^{30}$. 
Fig. 9 - La « vision sans regard » de Trombley

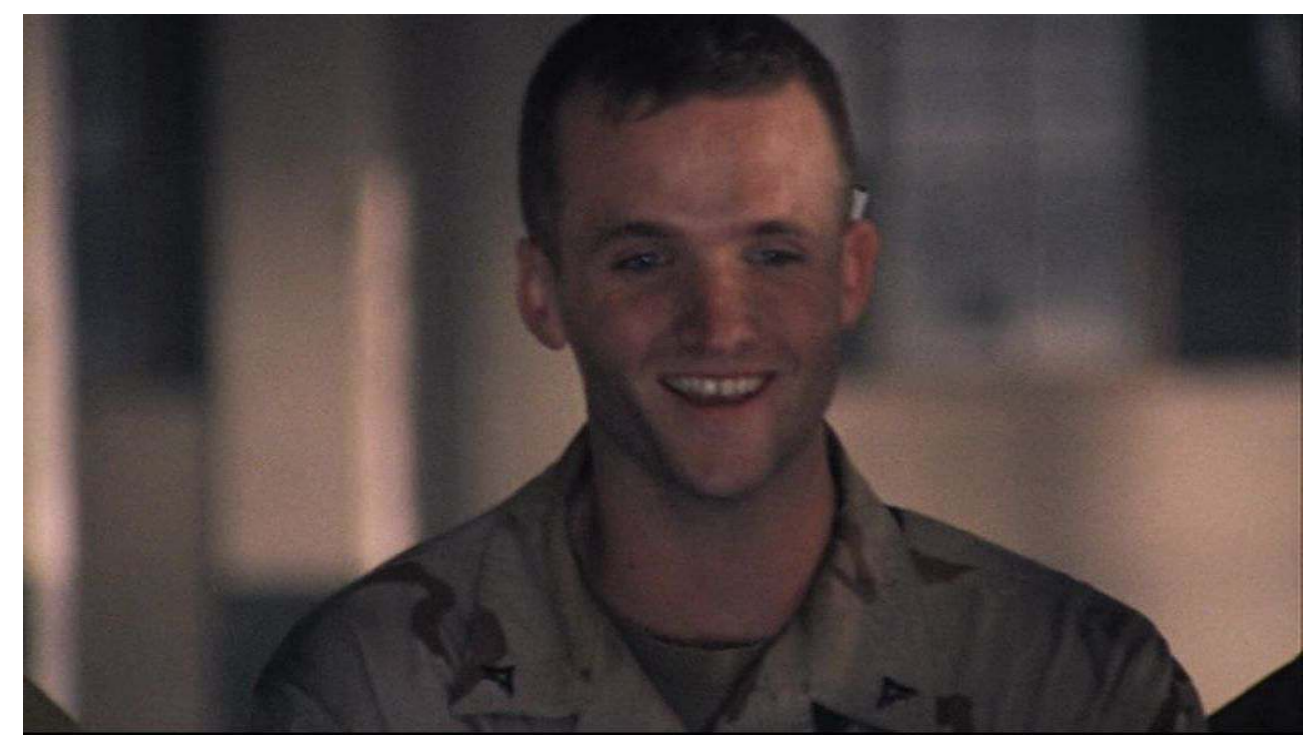

31 Mais l'intérêt principal de cette séquence est qu'elle invite à mesurer les effets de cette nouvelle modalité perceptive, en identifiant deux niveaux d'influence. Le premier concerne la perte de la syntaxe induite par la profusion des images. Parce qu'il est totalement dépourvu d'impact émotionnel sur la quasi-totalité des soldats, le montage vidéo de Lilley traduit leur incapacité à mettre en récit un conflit que, pourtant, ils vivent parfois dans leur chair. Le film amateur résulte d'une perception éclatée, juxtaposition de clichés dont est absent tout fil narratif et causal. Grâce à leurs prothèses audiovisuelles, les soldats reçoivent des images tellement nombreuses qu'elles découragent toute logique, des images qui ne racontent rien, ne témoignent de rien. À l'écran, l'horreur peut donc succéder à l'humour potache, et inversement, signe que tout est vécu comme une fiction, ou comme une boutade sans conséquences ${ }^{31}$.

32 Le deuxième domaine sur lequel influe l'abondance d'images privées de leur sens est l'individu dans sa dimension narcissique. Le film de Lilley constitue en effet un témoignage brut, focalisé sur l'expérience sensorielle des soldats, sur la réduction de l'événement à l'acte d'appuyer sur la détente, sur la joie de pouvoir dire «j'ai abattu quelque chose comme un vrai Marine ", sans s'apercevoir que le tir n'était pas plus réel que dans une salle d'arcade, mais que son effet, lui, l'était. Plus que de la guerre, c'est de cette recherche de sensations fortes que les soldats désirent témoigner. Par des vidéos comme celle de Lilley, ils veulent surtout prouver au monde qu'ils ont bien ressenti le frisson du tir meurtrier. Abattre une cible, même indéterminée, devient un rite de passage, et le film de guerre, selon la vision qu'en propose Lilley, un film collégial tourné pour se souvenir des bons moments de rigolade de la guerre d'Irak (Fig. 4). Les soldats semblent alors avoir vécu la guerre en marge du conflit, comme cantonnés dans l'un de ces bien nommés "camps de vacances » du Club Méditerranée où l'on a l'impression d'être en terre étrangère, sans avoir pour autant le moindre contact avec la réalité du pays visité. Le fait qu'ils obtiennent les informations sur le déroulement des opérations en écoutant la BBC ou via Internet, mentionné plusieurs fois au fil des sept épisodes, atteste également d'une relation indirecte avec l'extérieur, qui commence où s'arrête leur unité32.

33 Cet enfermement, plutôt qu'une contrainte, est un comportement induit par l'omniprésence des prothèses visuelles, dont la fonction est justement de garder le 
territoire envahi à distance pour ménager une intervention sans conséquences concrètes autres que celles que l'on peut difficilement distinguer sur un écran radar, ou à travers la perception en deux dimensions des appareils de vision nocturne. Dans la séquence finale, le viseur du bazooka rejoint ainsi dans sa fonction celui de la caméra, pour montrer que les deux dispositifs recherchent le même but ${ }^{33}$ : focaliser le sujet sur lui-même, le faire fonctionner en vase clos, et faire en sorte qu'il se mette en scène pour lui-même et pour ceux de son groupe, sans se soucier pour autant de ce qui se passe autour de lui - du moins au-delà d'un rayon d'inaction défini par la perception directe.

Mais si les seuls vestiges du conflit irakien résident dans ce montage hétéroclite et égocentré, ruine d'un simulacre de guerre qui, comme l'a montré Baudrillard à propos de la guerre du Golfe, n'a peut-être pas eu lieu, comment déterminer ce qu'il y avait à voir ? Que s'est-il passé en Irak, et comment en témoigner? À l'issue d'une série aussi aporétique que l'était en son temps Hiroshima mon amour, le montage final intégré au dernier épisode synthétise les bribes de réponses que l'on peut apporter à cette question. En révélant la conjonction entre la mise à distance de l'acte guerrier et le souci permanent qu'a l'individu de se mettre en scène pour témoigner de son propre vécu, cette ultime séquence montre que ce qui empêche de voir et de montrer la guerre ne se limite pas aux milieux militaires. Elle exprime ainsi l'idée centrale de Generation Kill, qui est que le paradoxe de l'immontrable vient de notre culture visuelle, et qu'une réflexion sur cette dernière constitue un préalable indispensable au témoignage.

\section{3. «Les films ont été faits le plus sérieusement possible. » Culture visuelle contemporaine et fiction militaire engagée}

Comme Hiroshima mon amour l'avait fait en son temps, Generation Kill prend acte du fait que le film de guerre est à réinventer dans sa forme, sous peine de ne rien montrer. Plutôt que de tenter la gageure de filmer un conflit dématérialisé, la série s'interroge ainsi sur ce qui fait l'inéluctabilité de cette stratégie du regard sans objet à laquelle doivent se conformer les Marines. La force de Generation Kill réside dans cette capacité à inventer des solutions narratives pour contrer ce "déni de l'image pleine ", cette angoisse de la vision insoutenable et inconcevable. L'enjeu est donc de taille, puisqu'il s'agit de renouveler certains aspects de la fiction antimilitariste considérés comme périmés suite à l'évolution de notre culture visuelle.

\section{Fiction antimilitariste et transfert de l'absurde}

Le comique de l'absurde et les situations grotesques qu'utilise la série s'inscrivent implicitement dans la tradition du cinéma engagé contre la guerre (on pense à Full Metal Jacket [Kubrick, 1987] et à Dr Strangelove [Kubrick, 1964], mais surtout à Redacted [De Palma, 2007]). En complément, l'attente sans fin véritable et donc sans but participe de la stratégie narrative des romans du même genre, du Désert des Tartares (Buzzatti, 1940) au Rivage de Syrtes (Gracq, 1951). Le but de cette technique est de confronter personnages et lecteurs/spectateurs à une situation aberrante. Sur ce dernier point, la série possède un avantage évident sur le cinéma, une proximité plus grande avec le roman, pour reconstituer l'impression d'une dilatation temporelle propice à retranscrire l'inanité 
d'une guerre où l'ennemi, visible mais absent, n'est qu'un fantôme. Plutôt qu'à l'horreur de la guerre, c'est à l'aridité du quotidien militaire que nous assistons en premier lieu. L'ambiance ainsi créée est celle d'une émission de téléréalité sur la vie des soldats, ou celle d'un reportage en temps réel, ayant fait l'objet d'un montage rudimentaire avant d'être diffusé. Autre signe qui témoigne d'un transfert de régime scopique entre les soldats et les spectateurs : nous attendons, avec eux, qu'il se passe quelque chose, car la fiction l'exige, et nous sommes, comme eux, déçus de constater que l'action, quand elle se produit, se révèle soit décevante parce qu'elle est avortée trop rapidement, soit déconcertante tant elle est longue et floue. Generation Kill montre ainsi que la vigilance bloque toute velléité de mettre en récit le quotidien, militaire ou non. Elle dénonce ainsi une entreprise globale dont le but est de prouver qu'il n'y avait rien à voir en Irak, qu'il n'y a rien à en dire, et qu'il ne s'y est donc rien passé.

\section{Terrorisme et réel furtif}

Cependant, nous partageons plus avec les Marines que l'attente d'un événement qui ne se produit jamais concrètement. Generation Kill illustre également la correspondance qu'établit Virilio entre le regard militaire, dont la technologie sert à amplifier la perspicacité, et notre conception de la réalité, influencée par cette supposée nécessité de $l^{\prime}$ hyperperception ${ }^{34}$. Elle va cependant plus loin, en montrant que la réciproque est tout aussi vraie : à mesure que l'art de la guerre se fait extension de l'œil, le regard, au quotidien, entre dans le cadre d'une stratégie militaire. C'est ce que l'on peut constater dans l'atmosphère de méfiance généralisée et le souci permanent de percer les apparences qui caractérisent la guerre contre le terrorisme et l'état esthétique de l'après 11 septembre.

Dans ce contexte, tous les types d'ennemis, et non uniquement les terroristes, sont censés être difficilement identifiables. Cela nécessite la mise en place d'une technologie hyperperceptive intégrale, c'est-à-dire qui ne soit pas seulement destinée aux ennemis de l'État, mais à la population mondiale dans son ensemble. Dans la série mythique de Simon et Burns, The Wire (HBO, 2002-08), ce contexte menait à l'utilisation massive d'une surveillance présentée comme la panacée pour prévenir non seulement les actes terroristes mais tous types de crimes. Cependant, son inefficacité crasse menait au constat que l'état de guerre permanent, que ce soit contre la drogue ou contre le terrorisme, permettait surtout de légitimer l'espionnage massif des individus. Generation Kill étudie le même phénomène dans le champ militaire, en montrant que la nature même du conflit irakien permet aux autorités d'organiser un combat à distance, où la technologie de l'hypervision permet paradoxalement de ne pas faire de distinctions. Ici, à la différence de ce qui se produit dans un contexte de surveillance policière, nul besoin de profilage : les Marines abattent sans discernement des soldats en armes et des civils, au motif que, dans leur viseur télescopique, rien ne permet de distinguer les uns des autres. Tout comme le terrorisme justifie une surveillance massive, le conflit irakien semble nécessiter d'agir de loin, en tirant « dans le tas » par mesure de précaution.

Generation Kill élabore ainsi une critique méthodique de l'argument technologique en matière de conflit. La série invite les spectateurs à s'apercevoir que la technologie, derrière l'alibi d'une guerre rapide et propre, entretient le mythe d'un ennemi invisible, immatériel ou fictionnel, qu'il faut abattre sans savoir qui il est ou s'il est légitime de le faire, par prudence ${ }^{35}$. La série s'attaque donc à un lieu commun de la guerre contre le 
terrorisme comme de la bataille rangée, selon lequel la vision décuplée donne à coup sûr la victoire à celui des deux camps qui la possède.

Par ce biais, Generation Kill critique l'utilisation néfaste de certaines associations d'idées qui structurent la pensée occidentale, mais qu'il semble à présent nécessaire de remettre en question : l'idée que la technologie est synonyme de progrès, et la conviction qu'existe un lien de causalité systématique entre voir, savoir et pouvoir. La série montre en effet que nous transposons au quotidien une attitude perceptive héritée de la logistique militaire, qui justifie l'utilisation de la vision à distance par la nécessité d'une vigilance de tous les instants. La banalisation des prothèses oculaires épouse donc une modification du régime scopique du réel: nous sommes invités à lire un comportement comme une façade, susceptible d'abriter la préparation d'un complot.

41 Cette tentation démiurgique d'un nouveau genre est commune à la lutte contre le terrorisme et à la tenue des conflits modernes. La première part du principe que la surveillance, parce qu'elle est prétendument dotée d'ubiquité et de permanence, parvient à percer à jour les dissimulateurs. La seconde pose que, pour gagner une guerre traditionnelle, il faut voir l'ennemi, mais ne pas le montrer à celui qui doit l'abattre. Il s'agit donc de nier l'existence de «cette part de subjectivité interprétative toujours à l'œuvre dans l'acte du regard ${ }^{36} »$.

\section{Découplage du regard et aperception philosophique}

En conséquence de quoi, charge reste aux spectateurs de s'ériger de nouveau en sujets par leur regard critique. En effet, si Generation Kill met en place une interaction entre son propre régime scopique et celui de la réalité, c'est pour finalement la détruire, et nous inviter ainsi à mieux y regarder. La série participe ainsi d'une logistique de l'aperception, au sens philosophique du terme cette fois, en proposant aux spectateurs d'accéder à «la conscience ou la connaissance réflexive de cet état intérieur » qu'est la perception ${ }^{37}$. Elle propose pour cela une épiphanie subtile, résumée dans la séquence finale.

43 L'habituel adieu aux personnages fréquentés à longueur d'épisodes passe ici par un découplage entre leur vision altérée des conflits et celle des spectateurs. À mesure qu'apparaissent les images atroces, elles passent subrepticement de l'ordinateur portable de Lilley à notre propre écran. Bientôt, nous sommes seuls à regarder ces clichés insoutenables. La série nous emmène alors voir ce qu'on peut trouver derrière les images de cette vidéo amateur. Elle nous invite ainsi à comparer notre position spectatorielle à celle de Trombley, dont la distance extrême face à l'image lui permet de supporter l'insoutenable, et même d'en rire. C'est en fonction de cette distance que les outils médiatiques imposent au réel que l'on peut espérer rematérialiser un conflit devenu anesthétique, raison pour laquelle Baudrillard le qualifie d'inexistant.

\section{Le viseur pris pour cible}

44 Cette réflexion sur l'omniprésence du régime des images est le but ultime de la séquence finale, au terme de laquelle notre culture visuelle se définit par l'hyperperception qui, premièrement, rassure et déculpabilise, deuxièmement, justifie l'intervention préventive. La fin suggère donc que le problème de l'immontrable ne résulte pas tant des images elles-mêmes, ni de leur éventuelle nature de pur simulacre, que du refus d'admettre que leur nature a changé. 


\begin{abstract}
précédent par la profusion des représentations du réel, la déconnexion des clichés entre eux par absence de mise en récit, le refus de toute structure logique qui risquerait d'y injecter du sens, la vitesse (ici, du montage, qui donne peu de poids aux contenus qu'il faut saisir au vol), la méfiance qui induit une distance esthétique systématique (résumée ici dans le plan d'un Saddam Hussein qui meurt comme statue, anticipation fictionnelle de sa mort effective annoncée [Fig. 10]), le narcissisme (puisque toute image, même documentaire, même militaire, témoigne avant tout de l'expérience de l'individu, qui l'utilise pour se construire une identité factice à l'écran), l'érotisation de la mise en scène qui suffit ici à provoquer la jouissance, la précision chirurgicale que traduit une obsession de la haute définition à la limite de l'obscène, la négation de l'espace qui fait que, d'un plan au suivant, on peut franchir des milliers de kilomètres en $1 / 10$ de seconde, et
\end{abstract}

de celle qui prévalait à l'époque de Hiroshima mon amour. Elle définit cette culture sans l'illusion de surpuissance liée à cette ubiquité.

Fig. 10 - La guerre d'Irak comme simulacre

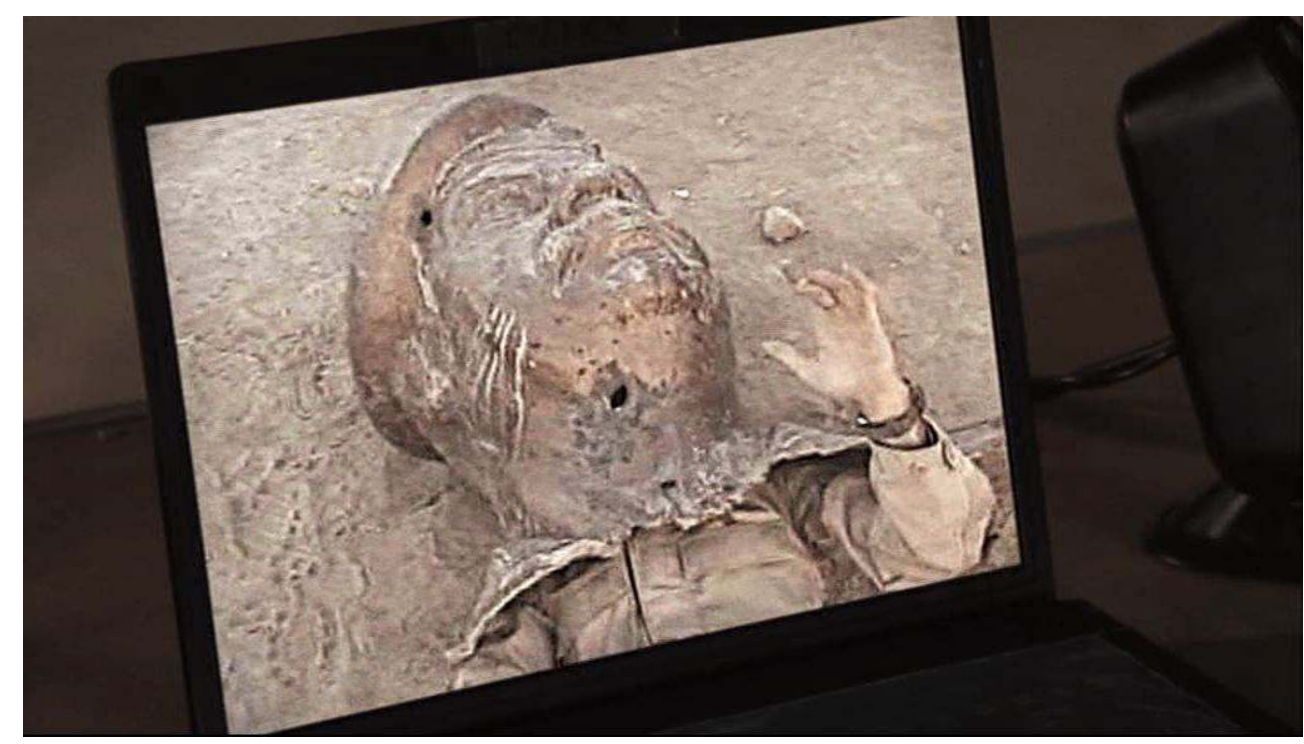

La chanson de Johnny Cash utilisée comme fond sonore, When the Man Comes Around, souligne ce dernier aspect, prométhéen, de notre culture. En plus d'inviter les spectateurs à se focaliser sur les éléments visuels en gommant les dialogues, ce texte introduit la problématique du jugement dernier et, plus généralement, pose la question des critères de discrimination entre les individus à travers l'évaluation de leurs actes ${ }^{38}$. Il nous place en position de juger, comme Dieu, en fonction de ce qu'on a vu, et de choisir, parmi les personnages, ceux qui mériteraient d'être sauvés. Résoudre cette question, cependant, relève de l'impossible. Malgré l'injonction à voir de nos propres yeux que nous transmet le texte ( behold $\left.{ }^{39} »\right)$, nous sommes témoins que les soldats pouvaient difficilement s'apercevoir de ce qu'ils faisaient.

\title{
Conclusion : aveugles de trop voir
}

Une société où tout le monde voit tout le monde nie la notion même de jugement dernier ${ }^{40}$. Grâce à une méthode critique originale, Generation Kill situe l'origine de ce vide 
épistémologique dans la culture visuelle contemporaine, en soulignant l'impact de la guerre contre le terrorisme sur la guerre d'Irak. Elle met en place une réflexivité d'un nouveau type, puisqu'elle aborde de front la mise à distance du réel par la technologie, effet distanciant caractéristique du régime scopique contemporain.

L'essence de notre culture visuelle est le seul élément dont nous puissions juger. En caractérisant notre rapport à l'image, dans cette séquence ultime comme dans l'ensemble des épisodes, Generation Kill nous ouvre donc les yeux sur notre configuration scopique, et redéfinit notre être au monde. La série montre que l'aporie du film de guerre n'est plus tant liée à la nature même des conflits, où l'événement se dissout dans l'explosion des bombes et se fait inexprimable, qu'à une culture visuelle qui montre tout et empêche ainsi de voir ${ }^{41}$.

Generation Kill propose ainsi un constat sur les modalités du «mensonge organisé » proche de celui que fait John Corner, pour qui il est réducteur de l'envisager simplement à la lumière du concept de propagande ${ }^{42}$. La série permet en effet d'envisager une manipulation à grande échelle qui utiliserait la culture visuelle pour induire des comportements. Cette stratégie étant avant tout sensorielle, elle se défend, du moins en apparence, de promouvoir certaines idées. La série montre que, si mensonge organisé il y a, c'est sur une logistique perceptive qu'il repose. Pour restaurer ce qui pourrait s'apparenter à une vérité, elle met en place une vision excentrique nécessaire et salutaire, puisque les médias, comme les armes, font en sorte que notre regard soit aligné sur une perspective prévue d'avance. C'est à ce prix que l'on peut retrouver une certaine incrédulité face à l'image ${ }^{43}$.

Si nous n'avons rien vu en Irak, c'est donc parce que nous étions comme Trombley, aveugles de trop y voir, insensibles à force de vouloir trop ressentir. À l'issue de Generation Kill, nous avons la possibilité d'adapter notre régime visuel à cette nouvelle essence de la représentation, comme Colbert qui acquiert la capacité de se rendre compte de ce qu'il a fait, ou de ce qu'il a fait faire à ceux qui étaient sous ses ordres. Le vrai combat contre l'horreur de la guerre se joue donc au quotidien, face aux images qui nous immergent dans un réel trop-plein, labyrinthe dont le théâtre des opérations n'est plus qu'un lieu sans spécificité.

\section{BIBLIOGRAPHIE}

AMMOUR-MAYEUR Olivier, « Témoigner de l'intémoignable. Hiroshima entre "remembrance" et vestiges de la mémoire (sur Hiroshima mon amour et Pluie noire) », in Interférences littéraires, nouvelle série, $\mathrm{n}^{\circ}$ 1, nov. 2008, « Écritures de la mémoire. Entre témoignage et mensonge », éd. David Martens et Virginie Renard, p. 133-144. http://interferenceslitteraires.be/sites/ drupal.arts.kuleuven.be.interferences/files/IL1\%20-\%20Ammour-Mayeur\%20-\%202008.pdf, consulté le 11 mai 2015.

CORNER, John, « Mediated politics, promotional culture and the idea of propaganda », Media Culture Society 29 (4), 2007, p. 669-77. 
LEIBNIZ, Gottfried Wilhelm, Principes de la nature et de la grâce, Paris, Charpentier, 1842.

NINEY, François, L'Épreuve du réel à l'écran : Essai sur le principe de réalité documentaire, Bruxelles, De Boeck, 2004.

SALMON, Christian, Storytelling. La machine à fabriquer des histoires et à formater les esprits, Paris, La Découverte, 2007.

SHAPIRO, Michael J., War Crimes, Atrocity, and Justice, Cambridge, RU, Polity Press, 2015.

VIRILIO, Paul, Guerre et cinéma I : Logistique de la perception (Nouvelle édition augmentée de l'édition de 1984), Paris, Cahiers du cinéma, 1991.

\section{NOTES}

1. «À partir [des bombardements d'Hiroshima et de Nagasaki], témoigner et se taire deviennent et l'un et l'autre inévitables, inconciliables, incompossibles, mais reflets l'un de l'autre dans un jeu d'emboîtement infini entre dire l'événement et l'impossibilité intrinsèque de le faire. Témoigner, mais de quoi? Comment témoigner? À qui et pour qui témoigner?» Olivier Ammour-Mayeur, «Témoigner de l'intémoignable. Hiroshima entre "remembrance” et vestiges de la mémoire (sur Hiroshima mon amour et Pluie noire) ", in David Martens et Virginie Renard (dir.) Interférences littéraires, nouvelle série, $\mathrm{n}^{\circ} 1$, nov. 2008 «Écritures de la mémoire. Entre témoignage et mensonge ", p. 133. http://interferenceslitteraires.be/sites/ drupal.arts.kuleuven.be.interferences/files/IL1\%20-\%20Ammour-Mayeur\%20-\%202008.pdf ， consulté le 11 mai 2015.

2. Ammour-Mayeur (Résumé de l'article).

3. Ammour-Mayeur, p. 137.

4. Ammour-Mayeur fait une analyse du « tout » figurant dans la réplique qui aboutit à un constat similaire. Ammour-Mayeur, p. 135.

5. Ammour-Mayeur, p. 137.

6. Le vocabulaire de la perception oculaire est d'ailleurs très fréquemment employé dans les dialogues de la série, ce qui vient renforcer l'impression d'une prédominance du visuel suscitée par la technologie. Ainsi, pour ne prendre que quelques exemples, le temps consacré au sommeil est qualifié de « shut eye », et repérer l'ennemi consiste à « poser les yeux » sur lui (" get eyes on them »).

7. Paul Virilio, Guerre et cinéma I: Logistique de la perception (Nouvelle édition augmentée de l'édition de 1984), Paris, Cahiers du cinéma, 1991, p. I. (Les indications en chiffres romains renvoient à la préface de l'édition de 1991).

8. Ibid.

9. Michael J. Shapiro propose une analyse similaire à propos du sergent Brad Colbert, personnage principal de la série dont la vision du conflit est médiée par la technologie perceptive qui équipe l'arsenal militaire, et en particulier les Humvees. Michael J. Shapiro, War Crimes, Atrocity, and Justice, Cambridge (R.U.), Polity Press, 2015, p. 88.

10. Virilio évoque « l'image de ce que ne cache plus la nuit». Virilio, p. IV.

11. Les nombreux gros plans sur les cartes d'état-major montrent bien que l'un des enjeux principaux du conflit est de se positionner par rapport à l'ennemi, tout en déterminant où il se trouve pour organiser un rapprochement relatif, qui se soldera par une intervention à distance.

12. Voir Shapiro, p. 89.

13. C'est ce que suggèrent, dans la séquence, les photos de jeunes femmes dénudées que les soldats accrochent dans leurs tentes. L'esthétique du dévoilement qui parcourt la série ravive en 
effet ce cliché de la fiction militaire, en l'ancrant dans un contexte où la possibilité de tout voir est primordiale.

14. Virilio, p. IV.

15. Héritée de la lutte contre le terrorisme, cette obsession pour la nécessité de débusquer l'ennemi vire ici à l'absurde, grâce à la trame narrative qui, dans les deux premiers épisodes, concerne la moustache de certains soldats. Bien que son port soit d'abord toléré, Schwetje l'interdit soudain. Cela permettra, selon lui, de repérer un éventuel ennemi caché parmi les troupes. Le souci permanent du profilage préventif mène ainsi au délit de faciès généralisé.

16. Shapiro a relevé l'absurdité d'une situation où les soldats, barricadés dans leurs chars d'assaut ou leurs Humvees, ne disposent pas de la moindre fenêtre sur l'extérieur. Leur contact sensoriel avec ce qui les entoure se résume donc essentiellement à ce qui transite par leurs appareils de vision à distance, qui s'apparentent alors à des périscopes, tant ils semblent établir le lien entre deux espaces fondamentalement différents (Shapiro, p 98). L'utilisation massive des plans d'ensemble à l'échelle de la série transcrit également cette perception qui se fait systématiquement à distance, et appelle l'utilisation d'un outil visuel pour se rapprocher de ce que l'on peine à discerner.

17. Quand elle n'est pas visuelle, la communication se fait également à distance. C'est ce que rappellent les échanges par radio qui servent de bande-son aux génériques de début et de fin mais aussi, dans l'ensemble des épisodes, l'intrusion dans le jargon militaire d'expressions empruntées à la communication par radio («Copy that » ou «Roger that " pour «c'est bien compris », par exemple).

18. LOVELL. Sir, danger-close is an artillery strike within six hundred meters of a friendly position. (Épisode 3).

19. Virilio décrit à ce propos « l'actuelle vidéosurveillance du champ de bataille » Virilio, p. I

20. Un élément de dialogue récurrent dans la série oppose Trombley, qui veut abattre des chiens, et son supérieur Colbert, qui lui rétorque que ça n'est pas en cela que consiste la guerre.

21. François Niney, L'Épreuve du réel à l'écran : Essai sur le principe de réalité documentaire, Bruxelles, De Boeck, 2004, p. 92.

22. Les règles à appliquer au combat prennent, dans les ordres qu'assène le lieutenant Nathaniel Fick, la forme d'un « catch 22 » visuel. D'une part, il enseigne aux soldats qu'il est légitime de tirer si on a l'impression de protéger son camp en le faisant. D'autre part, il rappelle que les tireurs potentiels incluent à la fois des gens en uniforme et des individus " habillés comme des paysans ». En guise de troisième temps de ce syllogisme incomplet, on peut donc déduire que les soldats doivent tirer sur tout ce qui bouge, et que le pouvoir de décision qui leur est laissé n'est qu'une illusion. Les cibles étant indifférenciées, on tire sans discrimination, pour s'apercevoir parfois qu'on a tiré sur des soldats de son propre camp. Le concept de légitime défense apparaît donc comme instable, ce qui n'est guère étonnant si l'on se remémore que la définition en varie d'un État américain à un autre et que, en Floride par exemple, le simple fait de «se sentir menacé » justifie le recours à la force.

23. L'expression « sensory overload » est prononcée dans l'épisode 7.

24. Sur les allusions à l'univers du jeu vidéo dans la série, voir Shapiro, p. 92.

25. Christian Salmon, Storytelling : La machine à fabriquer des histoires et à formater les esprits, Paris, La Découverte, 2007, p. 141 sq.

26. Pour ne prendre que quelques exemples : le sergent Brad Colbert est surnommé « Iceman », le lieutenant-colonel Stephen Ferrando est surnommé «Godfather », le sergent John Sixta reçoit plusieurs fois le surnom de «Mr. Potato Head», et le capitaine Dave McGraw celui de «Captain America ».

27. Salmon, p. 44 et p. 156. 
28. Dans ce nouveau contexte esthétique, « viser sans arrêt, ne plus se perdre de vue, c'est gagner ». Virilio, p. II. La conséquence en est que « voir et prévoir tendent alors à se confondre » Virilio, p. IV.

29. Shapiro émet un avis similaire sur la série : op cit., p. 91-92.

30. Virilio, p. III.

31. Sur les filtres filmiques, voir Shapiro, p. 95 sq.

32. Les répliques montrant que les informations provenant de l'extérieur sont obtenues par des voies indirectes sont nombreuses, et font souvent l'objet de plaisanteries comme celle, au début de la série, qui consiste à faire croire à Person que Jennifer Lopez vient de mourir. Preuve d'une coupure totale avec le monde extérieur : il a toutes les peines du monde à vérifier ou à infirmer cette information qui, si elle était fondée, ferait la une de tous les journaux.

33. La caméra est un « appareil de visée indirecte». Virilio, p.I.

34. Virilio, p. 71 sq.

35. Attitude que l'on peut rapprocher de la logique préventive qui caractérise de nombreux articles du USA PATRIOT Act du 26 novembre 2001: https:// www.gpo.gov/fdsys/pkg/ PLAW-107publ56/pdf/PLAW-107publ56.pdf .

36. Virilio, p. III.

37. Gottfried Wilhelm Leibniz, Principes de la nature et de la grâce, Paris, Charpentier, 1842, p. 407.

38. "There's a man going round taking names / And he decides who to free and who to blame. Everybody won't be treated all the same / There'll be a golden ladder reaching down / When the man comes around. »

39. Le texte de la chanson cite l'Apocalypse, $6: 12$ : «And I heard as it were the noise of thunder / One of the four beasts saying come and see and I saw / And behold a white horse»

40. Virilio parle d'une "volonté d'illumination généralisée, capable de tout donner à voir, à savoir, à chaque endroit, à chaque instant, version technicienne de l'œil de Dieu qui interdirait à jamais l'accident, la surprise. » Virilio, p. V.

41. Pour y remédier, il faut donc «investir dans la dissimulation " et mettre en place une « censure sur ces technologies» destinées à fournir des informations visuelles. Virilio, p.V.

42. John Corner, «Mediated politics, promotional culture and the idea of propaganda ", Media Culture Society 29 (4), 2007, p. 669-77.

43. Le récent Good Kill d'Andrew Niccol (2014) comporte un grand nombre de similitudes avec la série de Simon et Burns. La principale est que le personnage central de ce film se dissocie de la vision à distance des conflits pour se reconstruire une éthique par-delà l'impasse esthétique où l'accule son métier. Ancien pilote de chasse reconverti comme pilote de drones suite à la prolifération de ce nouveau type d'armes, il a pour rôle d'abattre, depuis une base de Las Vegas, des Talibans vivant à plusieurs milliers de kilomètres, et désignés comme cibles par ses supérieurs. En proie à sa conscience et désireux de se racheter pour ce qu'il considère comme des crimes, il ne trouve finalement d'autre moyen de le faire qu'à distance, scellant ainsi le succès d'une stratégie militaire qui dissocie l'acte guerrier de ses conséquences concrètes. Contrôlé en temps réel par sa hiérarchie, il lui est impossible de décider individuellement qui ne pas tuer, et ce d'autant moins que la vision surplombante de ses cibles les rend difficilement identifiables. En conséquence de quoi, son ultime geste d'impuissance consiste à profiter de quelques instants sans surveillance pour abattre un individu que ses supérieurs ne lui ont pas désigné : un Taliban qu'il a vu à de nombreuses reprises se rendre coupable de viol. Par cet acte de résistance pathétique, il atteste que les drones, et l'exécution à distance, imposent au comportement moral un cadre restrictif où le meurtre est le seul moyen d'expier le meurtre. 


\section{RÉSUMÉS}

Comme l'a montré Paul Virilio, la guerre repose depuis le premier conflit mondial sur une "véritable logistique de la perception militaire ». Je montre ici qu'en plus de confirmer le poids de la technologie dans les guerres modernes, l'omniprésence des appareils optiques dans Generation Kill dévoile la face cachée du dispositif martial, en insistant sur la mise en place d'une logistique de l'aperception. Ce phénomène, complémentaire de celui que décrit Virilio, est aussi plus récent. Il consiste à faire en sorte, toujours par technologie interposée, que l'adversaire soit au mieux aperçu, et au pire a-perçu, au sens où les Marines sont consciencieusement privés de leur faculté à en percevoir directement la présence.

La force de Generation Kill réside ainsi dans sa capacité à inventer des solutions narratives et visuelles pour témoigner de l'horreur des combats en dépit d'une technologie qui les met à distance. Plutôt que de tenter la gageure de filmer un conflit dématérialisé, la série s'interroge sur ce qui rend inéluctable la stratégie du regard sans objet à laquelle doivent se plier les Marines. Comme Hiroshima mon amour l'avait fait en son temps, Generation Kill prend acte que le film de guerre est à réinventer dans sa forme, sous peine de ne rien montrer. Pour ce faire, la série offre à ses spectateurs une logistique de l'aperception, au sens philosophique du terme cette fois, en leur proposant d'accéder à « la conscience ou [à] la connaissance réflexive de cet état intérieur » qu'est la perception.

Elle suggère que rendre compte des guerres modernes implique de se concentrer sur la vision, pour dénoncer l'hypocrisie technologique qui consiste, sous prétexte de décupler l'acuité visuelle des soldats, à cultiver l'invisibilité de l'ennemi. Grâce à une méthode critique originale, Generation Kill situe l'origine de ce vide entretenu dans la culture visuelle contemporaine, en soulignant l'impact de la guerre contre le terrorisme sur la campagne irakienne.

As Paul Virilio has argued, warfare has relied since the First World War on a "logistics of military perception". My argument in this paper is that Generation Kill does more than merely confirm the huge part played by technology in modern warfare. In addition, the series focuses on the omnipresence of optical devices on and around soldiers to reveal the dark side of the military apparatus by exposing the implementation of a logistics of aperception. This recent development comes as a complement to the one described by Virilio. Still by using technology as a go-between, aperception allows the foes to be at best seen in the distance, and at worst un-seen, which means that the Marines are meticulously deprived of their ability to perceive the enemy.

Generation Kill's critical scope thus lies in its ability to devise both narrative and visual solutions, as a fictional program, in order to bear witness to the horror of combat in spite of a technology that constantly puts violence at (more than) arm's-length. Rather than pursuing the impossible challenge of filming a conflict that has become immaterial, the series identifies what motivates the inevitably objectless gaze that characterizes the Marines it depicts. The same as Hiroshima mon amour had invented a way of dealing with nuclear warfare, Generation Kill acts on the observation that war movies must be reinvented, lest they should show nothing at all.

To achieve this goal, the series submits its viewers to a logistics of aperception, in the philosophical meaning of the term. Indeed, it enables them to access the "conscience or reflexive awareness of [perception as an] internal state". Generation Kill suggests that it is central to focus on vision in order to report on modern warfare. It thus exposes the current technological 
hypocrisy, which maintains the invisibility of the enemy under the pretense of amplifying the perceptual accuracy of soldiers. Thanks to its original critical method, Generation Kill locates the origin of the sustained emptiness of the gaze in contemporary visual culture, by emphasizing the impact of the war on terror on the Iraqi conflict.

INDEX

Mots-clés : Simon David, technologie, regard, terrorisme, guerre, Generation Kill

Keywords : War on Terror, technology, gaze

\section{AUTEUR}

\section{SÉBASTIEN LEFAIT}

Professeur d'études américaines et médiatiques à l'Université de Paris 8.

Ses travaux portent sur l'interaction entre le réel et ses représentations, à la frontière de la civilisation américaine, de la sociologie et des cultural studies, mais aussi des études théâtrales, cinématographiques et télévisuelles. 